\title{
On a System of Integrodifferential Equations Occurring in Reactor Dynamics
}

\section{J. J. LEVIN ${ }^{1} \&$ J. A. NOHEL}

Communicated by E. HopF

1. Introduction. In this paper we study the existence, uniqueness, and asymptotic behavior (as $t \rightarrow \infty$ ) of the solution of the real system

$$
\frac{d u}{d t}=-\int_{-\infty}^{\infty} \alpha(x) T(x, t) d x, \quad a \frac{\partial T}{\partial t}=b \frac{\partial^{2} T}{\partial x^{2}}+\eta(x) u \quad(0 \leqq t<\infty),
$$

satisfying the initial condition

$$
u(0)=u_{0}, \quad T(x, 0)=f(x) \quad(-\infty<x<\infty) .
$$

In (1.1), (1.2) $u=u(t), T=T(x, t)$ are the unknown functions, $\alpha, \eta, f$ are given real-valued functions, $u_{0}$ is a given real constant, and $a, b$ are given positive constants.

Of particular interest also is the "degenerate system" (obtained from (1.1) by setting $b=0$ )

$$
\frac{d u}{d t}=-\int_{-\infty}^{\infty} \alpha(x) T(x, t) d x, \quad a \frac{\partial T}{\partial t}=\eta(x) u \quad(0 \leqq t<\infty),
$$

where $u, T$ satisfy the initial condition (1.2) and where $\alpha, \eta, a$ are the same as in (1.1). We investigate the relationship of the solution of (1.1), (1.2) to that of (1.3), (1.2) as $b \rightarrow 0+$.

The system (1.1) describes the linearized dynamic model of a continuous medium nuclear reactor. The interpretation of the various quantities in (1.1) is

$t \quad=$ time,

$x \quad=$ position along the reactor (regarded as a doubly infinite rod),

$u(t)=$ logarithm of the total reactor power $(u(t) \equiv 0$ at equilibrium),

$T(x, t)=$ deviation of the temperature from equilibrium $(T(x, t) \equiv 0$ at equilibrium),

\footnotetext{
${ }^{1}$ Supported by U.S. Army, Navy, and Air Force under Air Force Contract AF 19(604)-5200.

2 Supported by U. S. Army under Contract No. DA-11-022-ORD-2059, and in part by the National Science Foundation, N.S.F. G-7343.
} 


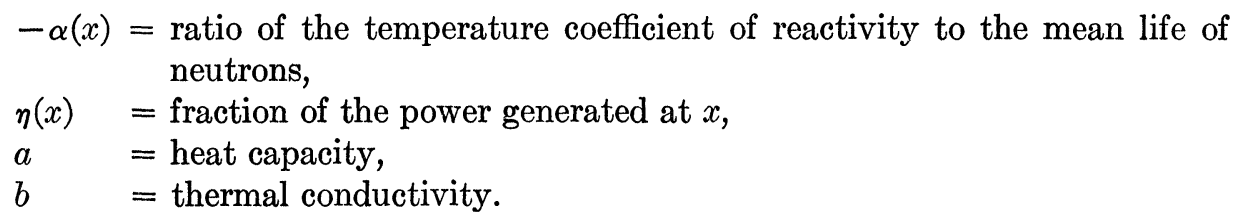

For general remarks about the dynamics of various reactor models and physical assumptions we refer to Ergen [1]. We note, however, that in the nonlinear dynamic model of a continuous medium nuclear reactor the term $\eta(x) u$ in (1.1) is replaced by $\eta(x)\left(e^{u}-1\right)$. Stability questions for the nonlinear problem have been considered (heuristically) by ERGEN \& NoHeL [2]; they combine Liapounov's Second Method with physically plausible arguments that have not been justified rigorously. The present results (in particular, Theorem 2) imply that the equilibrium solution $u(t) \equiv 0, T(x, t) \equiv 0$ of $(1.1)$ is asymptotically stable in the sense that the solution $\{u(t ; b), T(x, t ; b)\}$ of (1.1), (1.2) approaches zero as $t \rightarrow \infty$ (uniformly in $x,-\infty<x<\infty$ ).

The continuous medium model may be regarded as a limiting case of a heterogeneous reactor consisting of $n$ media. A rigorous discussion of stability for such reactors and other relatively simple reactor models involving nonlinear ordinary differential equations is contained in a recent paper of ERGEN, LIPKIN $\&$ NoHer [3].

In certain practical considerations of the continuous medium reactor it is advantageous to make use of the "degenerate system" (1.3) rather than (1.1). The hope here is, of course, that the extreme smallness of $b$ will cause solutions of (1.3) to be good approximations of solutions of (1.1). It will be shown, Theorem 5 , that this procedure is valid only over a finite time interval. This situation is reminiscent of that occurring in singular perturbation problems; however, here the bad behavior occurs at $t=\infty$ and not at, say, an initial boundary layer.

The hypotheses of our theorems, see Section 2, are quite general from a physical point of view. In a reactor one can always assume that $\alpha, \eta, f$ are bounded and vanish outside a finite interval; thus (2.1), (2.2), (2.4), (2.7) are not at all physically restrictive. The smoothness conditions (2.3), (2.5) are physically reasonable and are used in the application of the classical theory of the heat equation. One would also always have $\alpha(x) \geqq 0, \eta(x) \geqq 0$ and $\alpha(x) \neq 0, \eta(x) \neq 0$; thus if one makes the further (physically reasonable) assumption that $\alpha(x)$ is continuous, it follows that (2.8) is satisfied. From Theorem 4 one sees that (2.9) is necessary for the degenerate problem (1.3), (1.2) to have a periodic solution; this is the only case of physical interest. The physically interesting conditions $\eta(x)=k \alpha(x), k$ a positive constant, and $\alpha(x) \neq 0$ on a set of positive measure are seen to imply hypothesis (2.6). Another physically natural set of conditions which implies (2.6), by an easy application of Section 68 of ACHIESER [4, pp. 125-126], is: $\alpha, \eta$ even; $(-1)^{k} \alpha^{(k)}(x) \geqq 0,(-1)^{k} \eta^{(k)}(x) \geqq$ $0(k=0,1,2 ; 0 \leqq x<\infty) ; \alpha(x), \eta(x) \neq 0$; and (2.2). It should be noted that (2.1), (2.6) imply (2.9) by the Parseval theorem. 
We wish to acknowledge several very stimulating conversations with our colleagues P. M. Anselone and H. F. Bueckner of the Mathematics Research Center and R. Prosser of Lincoln Laboratory.

2. Summary of Results. The following non-independent but consistent hypotheses will be used at various points:

$$
\begin{gathered}
\alpha, \eta, f \varepsilon L_{2}(-\infty, \infty), \\
\alpha, \eta, f \varepsilon L_{1}(-\infty, \infty), \\
\eta, f \varepsilon C(-\infty, \infty), \\
\sup _{-\infty<x<\infty}\{|\alpha(x)|,|\eta(x)|,|f(x)|\} \leqq K_{1}, \\
\left|\eta\left(x_{1}\right)-\eta\left(x_{2}\right)\right| \leqq K_{2}\left|x_{1}-x_{2}\right| \quad\left(-\infty<x_{1}, x_{2}<\infty\right), \\
\operatorname{Re}\{\hat{\eta}(x) \hat{\alpha}(-x)\} \geqq 0, \quad \operatorname{Re}\{\hat{\eta}(x) \hat{\alpha}(-x)\}>0
\end{gathered}
$$

on a set of positive measure $(0 \leqq x<\infty)$,

$$
\begin{gathered}
\alpha(x), \eta(x), f(x)=O\left(e^{-\lambda|x|}\right) \quad(\lambda>0 ;|x| \rightarrow \infty), \\
\left(\int_{-\infty}^{\infty} \alpha(x) d x\right)\left(\int_{-\infty}^{\infty} \eta(x) d x\right) \neq 0, \\
(\alpha, \eta)=\int_{-\infty}^{\infty} \alpha(x) \eta(x) d x>0,
\end{gathered}
$$

where the $K_{i}$ and $\lambda$ are positive constants, the $L_{i}$ are the usual Lebesgue classes, $C$ is the class of continuous functions, $\hat{\eta}(x)$ is the Fourier transform

$$
\hat{\eta}(x)=\mathscr{F}\{\eta(x)\}=\int_{-\infty}^{\infty} e^{-i x y} \eta(y) d y,
$$

similarly $\hat{\alpha}(x)$ and $\hat{f}(x)$ are the Fourier transforms of $\alpha(x)$ and $f(x)$, respectively, and $\operatorname{Re}\{z\}$ is the real part of $z$.

For ease of reference in the proofs, some redundancy in the statement of the hypotheses of the theorems has been made. For example, in Theorems 1, 3, and 5 note that (2.2), (2.4) imply (2.1); and in Theorem 2 note that (2.4), (2.7) imply (2.1) and (2.2).

Concerning the existence of a solution of (1.1), (1.2) we prove the following

Theorem 1. Let (2.1)-(2.5) be satisfied, Then there exists a solution $u(t ; b)$, $T(x, t ; b)$ of $(1.1),(1.2)$ on $-\infty<x<\infty, 0 \leqq t<\infty$.

It will be seen from the proof of Theorem 1 that some relaxing of the hypothesis is possible. Since our main interest is the asymptotic behavior of solutions, for which we require much stronger conditions, no attempt will be made to prove each result under the weakest possible assumptions. ${ }^{3}$

The principal result is

\footnotetext{
${ }^{3}$ See the appendix.
} 
Theorem 2. Let (2.1)-(2.8) be satisfied. Let $u(t ; b), T(x, t ; b)$ be the solution of (1.1), (1.2) of Theorem 1. Then there exist constants $d_{j}=d_{j}(b)(j=1,2, \cdots)$ such that

$$
\lim _{t \rightarrow \infty} t^{n}\left[u(t ; b)-\frac{1}{\pi t^{\frac{1}{2}}} \sum_{j=1}^{n}(-1)^{j} d_{j} \frac{\Gamma\left(j+\frac{1}{2}\right)}{t^{j}}\right]=0 \quad(n=1,2, \cdots),
$$

and, in particular,

$$
\lim _{t \rightarrow \infty} t^{\frac{3}{2}} u(t ; b)=-\frac{1}{2 \pi^{\frac{1}{2}}} d_{1}
$$

Furthermore,

$$
T(x, t ; b)=O\left(t^{-\frac{1}{2}}\right) \quad(t \rightarrow \infty, \text { uniformly in } x) .
$$

The constants $d_{1}$ and $d_{2}$ are given by the formulae

$$
\begin{aligned}
& \left\{\begin{array}{l}
d_{1}=u_{0} \frac{2(a b)^{\frac{1}{2}}}{H_{1}(0)}+\frac{a^{\frac{3}{2}}}{b^{\frac{1}{2}}}\left(\frac{H_{1}^{\prime}(0) H_{2}(0)-H_{2}^{\prime}(0) H_{1}(0)}{H_{1}^{2}(0)}\right) \\
d_{2}=u_{0} \frac{a^{\frac{3}{2}}}{b^{\frac{1}{2}}}\left(2 \frac{\left[H_{1}^{\prime}(0)\right]^{2}}{H_{1}^{3}(0)}-\frac{H_{1}^{\prime \prime}(0)}{H_{1}^{2}(0)}\right)+2 a^{\frac{3}{2}} b^{\frac{1}{2}} \frac{H_{2}(0)}{H_{1}^{2}(0)}
\end{array}\right. \\
& -\frac{1}{2} \frac{a^{5 / 2}}{b^{\frac{3}{2}}}\left(\frac{1}{3} \frac{H_{2}^{\prime \prime \prime}(0)}{H_{1}(0)}-\frac{H_{1}^{\prime}(0) H_{2}^{\prime \prime}(0)+H_{1}^{\prime \prime}(0) H_{2}^{\prime}(0)+\frac{1}{3} H_{1}^{\prime \prime \prime}(0) H_{2}(0)}{H_{1}^{2}(0)}\right. \\
& \left.+2 \frac{\left[H_{1}^{\prime}(0)\right]^{2} H_{2}^{\prime}(0)+H_{1}^{\prime}(0) H_{1}^{\prime \prime}(0) H_{2}(0)}{H_{1}^{3}(0)}-2 \frac{\left[H_{1}^{\prime}(0)\right]^{3} H_{2}(0)}{H_{1}^{4}(0)}\right),
\end{aligned}
$$

where

$$
\left\{\begin{array}{l}
H_{1}^{(n)}(0)=(-1)^{n} \int_{-\infty}^{\infty} \int_{-\infty}^{\infty}|x-y|^{n} \alpha(x) \eta(y) d x d y \\
H_{2}^{(n)}(0)=(-1)^{n} \int_{-\infty}^{\infty} \int_{-\infty}^{\infty}|x-y|^{n} \alpha(x) f(y) d x d y .
\end{array} \quad(n=0,1, \cdots)\right.
$$

Hypothesis (2.8) implies that $H_{1}(0) \neq 0$. We shall remark in Section 5 on an alternative to (2.8); however, we again remark that (2.8) is satisfied in the physically interesting cases. It might also be mentioned that a formula similar to $(2.10)$ with $d_{i}=d_{i}(x ; b)$ can be obtained for $T(x, t ; b)$ by the methods of Section 5 .

If $d_{1} \neq 0$ then from (2.11) it follows that the solution $u(t ; b)$ does not have zeros for arbitrarily large $t$, no matter how small $b>0$ is. In fact, as can easily be shown from (2.10), this is true as long as the $d_{i}$ do not all vanish. In view of Theorems 4 and 5 below, this result is somewhat surprising.

The next theorem gives a class of pairs of functions in which the solution $u(t ; b), T(x, t ; b)$ of Theorem 1 is unique. It is easily shown that (2.15), (2.16) are satisfied by $u(t ; b), T(x, t ; b)$. 
Theorem 3. Let (2.1)-(2.5) be satisfied. Then there is at most one solution $u(t)$, $T(x, t)$ of (1.1), (1.2) such that

$$
\begin{aligned}
& \int_{0}^{\infty}|u(t)| d t<\infty, \\
& \sup _{\substack{\infty<x<\infty \\
0 \leqq t<\infty}}|T(x, t)|<\infty .
\end{aligned}
$$

The degenerate problem (1.3), (1.2) is completely solved by

Theorem 4. Let (2.1) and (2.9) be satisfied. Then (1.3), (1.2) has the unique solution

$$
\left\{\begin{aligned}
v(t)=-\frac{(\alpha, f)}{\left[\frac{1}{a}(\alpha, \eta)\right]^{\frac{1}{3}}} \sin \left[\frac{1}{a}(\alpha, \eta)\right]^{\frac{1}{2}} t+u_{0} \cos \left[\frac{1}{a}(\alpha, \eta)\right]^{\frac{1}{2}} t \\
\pi(x, t)=f(x)+\frac{\eta(x)}{a}\left\{\frac{(\alpha, f)}{\frac{1}{a}(\alpha, \eta)}\left[\cos \left[\frac{1}{a}(\alpha, \eta)\right]^{\frac{1}{2}} t-1\right]\right. \\
\left.+\frac{u_{0}}{\left[\frac{1}{a}(\alpha, \eta)\right]^{\frac{1}{2}}} \sin \left[\frac{1}{a}(\alpha, \eta)\right]^{\frac{1}{2}} t\right\}
\end{aligned}\right.
$$

It has already been observed that (2.1), (2.6) imply (2.9); however, it will be seen that (2.9) arises naturally in Theorem 4 with no reference to (2.6).

The behavior of the solution $u(t ; b), T(x, t ; b)$ of Theorem 1 as $b \rightarrow 0+$ is described in

Theorem 5. Let (2.1)-(2.5) and (2.9) be satisfied. Let $u(t ; b), T(x, t ; b)$ and $v(t), \pi(x, t)$ be as in Theorems 1 and 4 respectively. Let $T_{1}$ be any constant satisfying $0 \leqq T_{1}<\infty$. Then

$$
\lim _{b \rightarrow 0+} u(t ; b)=v(t)
$$

uniformly over $0 \leqq t \leqq T_{1}$, and for each $x$ in $-\infty<x<\infty$

$$
\lim _{b \rightarrow 0+} T(x, t ; b)=\pi(x, t)
$$

uniformly over $0 \leqq t \leqq T_{1}$.

3. Formal Calculations. In this section we obtain, by purely formal means, a Volterra equation, (3.12), which is central to most of our analysis. The lemmas of Section 4 establish the existence and uniqueness (which are classic) together with some properties of the solution of (3.12), including the equivalence of (3.12) to (3.7) as well as to (3.10) (both with the initial condition $u(0)=u_{0}$ ), that are needed in the sequel. The asymptotic behavior of the solution of (3.12) is investigated in Section 5. 
Assume that (1.1), (1.2) has a solution $u(t), T(x, t)$. Let

$$
\begin{gathered}
G(x, t)=\left[4 \pi \frac{b}{a} t\right]^{-\frac{1}{2}} \exp \left\{-\frac{x^{2}}{4(b / a) t}\right\}, \\
T_{H}(x, t)=\int_{-\infty}^{\infty} G(x-\xi, t) f(\xi) d \xi, \\
\tilde{T}(x, t)=T(x, t)-T_{H}(x, t) .
\end{gathered}
$$

Then (1.1), (1.2) becomes

$$
\left\{\begin{array}{l}
\frac{d u}{d t}=-\int_{-\infty}^{\infty} \alpha(x) \tilde{T}(x, t) d x-\int_{-\infty}^{\infty} \int_{-\infty}^{\infty} G(x-\xi, t) f(\xi) \alpha(x) d \xi d x \\
a \frac{\partial \tilde{T}}{\partial t}=b \frac{\partial^{2} \tilde{T}}{\partial x^{2}}+\eta(x) u, \\
u(0)=u_{0}, \quad \tilde{T}(x, 0) \equiv 0 \quad(-\infty<x<\infty) .
\end{array}\right.
$$

Assuming that

$$
\tilde{T}(x, t)=\frac{1}{a} \int_{0}^{t} \int_{-\infty}^{\infty} G(x-\xi, t-\tau) \eta(\xi) u(\tau) d \xi d \tau,
$$

which formally satisfies the second equation of (3.4) and the second initial condition of (3.5), then the first equation of (3.4) becomes

$$
\begin{aligned}
\frac{d u}{d t}=-\frac{1}{a} \int_{0}^{t}\left\{\int_{-\infty}^{\infty} \int_{-\infty}^{\infty} G(x-\xi, t\right. & -\tau) \eta(\xi) \alpha(x) d \xi d x\} u(\tau) d \tau \\
& -\int_{-\infty}^{\infty} \int_{-\infty}^{\infty} G(x-\xi, t) f(\xi) \alpha(x) d \xi d x
\end{aligned}
$$

Equation (3.7) can be greatly simplified by employing the Parseval and convolution theorems for Fourier transforms. Thus, noting that

we obtain

$$
\mathscr{F}\left\{\frac{\exp \left\{-x^{2} / \rho\right\}}{(\pi \rho)^{\frac{1}{2}}}\right\}=\exp \left\{-\frac{\rho}{4} x^{2}\right\} \quad(\rho>0),
$$

$$
\begin{aligned}
\frac{d u}{d t}=-\frac{1}{2 \pi a} \int_{0}^{t}\left\{\int_{-\infty}^{\infty} \exp \left\{-\frac{b}{a} x^{2}(t-\tau)\right\} \hat{\eta}(x) \hat{\alpha}(-x) d x\right\} u(\tau) d \tau \\
-\frac{1}{2 \pi} \int_{-\infty}^{\infty} \exp \left\{-\frac{b}{a} x^{2} t\right\} \hat{f}(x) \hat{\alpha}(-x) d x
\end{aligned}
$$

It is easily seen, by an elementary change of variable, that (3.9) may be written as

$$
\begin{aligned}
\frac{d u}{d t}=-\frac{1}{\pi a} \int_{0}^{t}\left\{\int_{0}^{\infty} \exp \left\{-\frac{b}{a} x^{2}(t-\tau)\right\}\right. & \left.h_{1}(x) d x\right\} u(\tau) d \tau \\
& -\frac{1}{\pi} \int_{0}^{\infty} \exp \left\{-\frac{b}{a} x^{2} t\right\} h_{2}(x) d x
\end{aligned}
$$


where

$$
h_{1}(x)=\operatorname{Re}\{\hat{\eta}(x) \hat{\alpha}(-x)\}, \quad h_{2}(x)=\operatorname{Re}\{\hat{f}(x) \hat{\alpha}(-x)\} .
$$

Integrating (3.10) yields the Volterra equation

$$
\begin{aligned}
u(t)=u_{0}-\frac{1}{\pi a} \int_{0}^{t}\left\{\int_{0}^{\infty} \frac{1}{\frac{b}{a} x^{2}}\right. & {\left.\left[1-\exp \left\{-\frac{b}{a} x^{2}(t-\tau)\right\}\right] h_{1}(x) d x\right\} u(\tau) d \tau } \\
& -\frac{1}{\pi} \int_{0}^{\infty} \frac{1}{\frac{b}{a} x^{2}}\left[1-\exp \left\{-\frac{b}{a} x^{2} t\right\}\right] h_{2}(x) d x .
\end{aligned}
$$

4. Proof of Theorem 1. We first prove some lemmas concerning (3.12).

Lemma 4.1. Let (2.1) be satisfied. Then (3.12) has a unique solution $u(t ; b)$ for $0 \leqq t<\infty$. Moreover, $u^{\prime}(t ; b)$ exists and is continuous and

$$
u(t ; b), u^{\prime}(t ; b)=O\left(e^{\gamma t}\right) \quad(t \rightarrow \infty) \quad\left(\prime=\frac{d}{d t}\right)
$$

for some $\gamma=\gamma(b)>0$.

Proof. Define the real functions $h_{i}^{*}(t)(i=1,2)$ by

$$
\left\{\begin{array}{l}
h_{1}^{*}(t)=-\frac{1}{\pi a} \int_{0}^{\infty} \frac{1}{\frac{b}{a} x^{2}}\left[1-\exp \left\{-\frac{b}{a} x^{2} t\right\}\right] h_{1}(x) d x \\
h_{2}^{*}(t)=-\frac{1}{\pi} \int_{0}^{\infty} \frac{1}{\frac{b}{a} x^{2}}\left[1-\exp \left\{-\frac{b}{a} x^{2} t\right\}\right] h_{2}(x) d x
\end{array}\right.
$$

Then (3.12) becomes

$$
u(t)=u_{0}+\int_{0}^{t} h_{1}^{*}(t-\tau) u(\tau) d \tau+h_{2}^{*}(t) .
$$

Hypothesis (2.1), standard Fourier transform theory, and definition (3.11) imply that

$$
h_{i}(x) \varepsilon L_{1}(0, \infty) \quad(i=1,2),
$$

which, together with the elementary inequality

$$
0 \leqq \frac{1}{\frac{b}{a} x^{2}}\left[1-\exp \left\{-\frac{b}{a} x^{2} t\right\}\right] \leqq t \quad(0 \leqq x, t<\infty),
$$

implies that the $h_{i}^{*}(t)$ are continuous on $0 \leqq t<\infty$. This last fact is enough, by a well known theorem on Volterra equations, to guarantee the existence and uniqueness of a real solution $u(t ; b)$ of (4.3) (and, hence, of (3.12)). 
To complete the proof of the lemma we need to establish (4.1). It is well known, from the continuity of the $h_{i}^{*}(t)$, that the successive approximations

$$
\left\{\begin{aligned}
u_{0}(t) & =u_{0} \\
u_{n+1}(t) & =u_{0}+\int_{0}^{t} h_{1}^{*}(t-\tau) u_{n}(\tau) d \tau+h_{2}^{*}(t) \quad(n=0,1, \cdots)
\end{aligned}\right.
$$

converge to the solution $u(t ; b)$ for all $t$. In particular, one has that the series

$$
u(t ; b)=u_{0}(t)+\sum_{n=1}^{\infty} u_{n}(t)-u_{n-1}(t)
$$

converges uniformly to $u(t ; b)$ on every finite $t$ interval. From (4.2), (4.4), (4.5) there exists a constant $K_{3}>0$ such that

$$
\left|h_{i}^{*}(t)\right| \leqq K_{3} t \quad(i=1,2 ; 0 \leqq t<\infty) .
$$

An induction, using (4.6), (4.8), yields

$$
\left|u_{n}(t)-u_{n-1}(t)\right| \leqq K_{3}^{n}\left|u_{0}\right| \frac{t^{2 n}}{(2 n) !}+K_{3}^{n} \frac{t^{2 n-1}}{(2 n-1) !} \quad(n=1,2, \cdots),
$$

which, together with (4.7), implies

$$
|u(t ; b)| \leqq\left[\left|u_{0}\right|+K_{3}^{\frac{1}{2}}\right] \exp \left\{K_{3}^{\frac{1}{2}} t\right\} \quad(0 \leqq t<\infty) .
$$

To establish the order condition for the derivative we observe first that

$$
\left\{\begin{array}{l}
\frac{d h_{1}^{*}(t)}{d t}=-\frac{1}{\pi a} \int_{0}^{\infty} \exp \left\{-\frac{b}{a} x^{2} t\right\} h_{1}(x) d x \\
\frac{d h_{2}^{*}(t)}{d t}=-\frac{1}{\pi} \int_{0}^{\infty} \exp \left\{-\frac{b}{a} x^{2} t\right\} h_{2}(x) d x,
\end{array}\right.
$$

for by (4.4) the integrals converge uniformly with respect to $t$. From (4.2), (4.3), (4.11) it follows that

$$
u^{\prime}(t ; b)=\int_{0}^{t}\left\{\frac{d}{d t} h_{1}^{*}(t-\tau)\right\} u(\tau ; b) d \tau+\frac{d}{d t} h_{2}^{*}(t)
$$

and from (4.4), (4.8), (4.11) that

$$
\left|\frac{d h_{i}^{*}(t)}{d t}\right| \leqq K_{3} \quad(i=1,2 ; 0 \leqq t<\infty) .
$$

Hence, from (4.12), (4.13),

$$
\left|u^{\prime}(t ; b)\right| \leqq K_{3} \int_{0}^{t}|u(\tau ; b)| d \tau+K_{3} \quad(0 \leqq t<\infty) .
$$

From (4.10), (4.14) one easily has

$$
u^{\prime}(t ; b)=O\left(\exp \left\{K_{3}^{\frac{1}{3}} t\right\}\right) \quad(t \rightarrow \infty),
$$

which completes the proof of the lemma. 
From now on $u(t ; b)$ is the function defined in Lemma 4.1. As the notation anticipates, it is also the function of Theorem 1.

Lemma 4.2. Under the hypothesis of Lemma 4.1, (3.10), with the initial condition $u(0)=u_{0}$, is equivalent to (3.12). Thus $u(t ; b)$ is the only solution of (3.10) such that $u(0)=u_{0}$.

Proof. It has already been shown in the course of proving Lemma 4.1 that $u(t ; b)$ satisfies (3.10); see (4.11), (4.12) which is just another way of writing (3.10). Conversely, let $u(t)$ be a solution of (3.10) satisfying $u(0)=u_{0}$. Integration of (3.10) and use of Fubini's theorem (recall (4.4)) show that $u(t)$ is a solution of (3.12). Hence $u(t) \equiv u(t ; b)$, which completes the proof.

Lemma 4.3. Under the hypothesis of Lemma 4.1, (3.7), with the initial condition $u(0)=u_{0}$, is equivalent to (3.12). Thus $u(t ; b)$ is the only solution of (3.7) such that $u(0)=u_{0}$.

Proof. By Lemma 4.2 it suffices to establish the equivalence of (3.10) and (3.7). Note first that (3.9) is simply another way of writing (3.10). Then (2.1) and the convolution and Parseval theorems show that (3.7) is merely another way of writing (3.9). This completes the proof of the lemma.

To complete the proof of Theorem 1 define

$$
\left\{\begin{array}{l}
T_{H}(x, t ; b)=\int_{-\infty}^{\infty} G(x-\xi, t) f(\xi) d \xi \\
\tilde{T}(x, t ; b)=\frac{1}{a} \int_{0}^{t} \int_{-\infty}^{\infty} G(x-\xi, t-\tau) \eta(\xi) u(\tau ; b) d \xi d \tau, \\
T(x, t ; b)=T_{H}(x, t ; b)+\tilde{T}(x, t ; b),
\end{array}\right.
$$

where $u(t ; b)$ is the unique solution of (3.12). By the classical theory of the heat equation, see e.g. Rosenbloom [5; Ch. 4], using (2.3), (2.4), (2.5), it follows that $u(t ; b), T(x, t ; b)$ satisfy the second equation of (1.1) and of (1.2). Of course, $u(0 ; b)=u_{0}$ by $(3.12)$, so that the first equation of (1.2) is satisfied. Thus it only remains to show that the first equation of $(1.1)$ is satisfied by $u(t ; b)$, $T(x, t ; b)$. From (4.15) and $(2.2)$ we have

$$
\begin{aligned}
\int_{-\infty}^{\infty} \alpha(x) T(x, t & ; b) d x \\
=\frac{1}{a} \int_{0}^{t}\left\{\int_{-\infty}^{\infty} \int_{-\infty}^{\infty} G(x\right. & -\xi, t-\tau) \eta(\xi) \alpha(x) d \xi d x\} u(\tau ; b) d \tau \\
& \quad+\int_{-\infty}^{\infty} \int_{-\infty}^{\infty} G(x-\xi, t) f(\xi) \alpha(x) d \xi d x
\end{aligned}
$$

the interchange of order of integration being justified by Fubini's theorem. Comparing (4.16) and (3.7), with $u(t)$ replaced by $u(t ; b)$ in the latter (using Lemma 4.3), we see that the first equation of (1.1) is satisfied. This completes 
the proof of Theorem 1 . We remark that the boundedness of $\alpha(x)$ was not used in the proof and that (2.2) is not really essential here, though convenient. These assumptions are, however, used later.

5. Proof of Theorem 2. We shall first prove (2.10) which, in view of Section 4 , is really a theorem about the asymptotic behavior of the solution of the Volterra equation (3.12). In this connection we remark that the situation here is different from the one considered by FELLER [6] in studying the asymptotic behavior of solutions of the "Renewal Equation". For example, in the present case the functions $h_{1}^{*}(t)$ and $u_{0}+h_{2}^{*}(t)$ are not integrable over $(0, \infty)$, nor are they nonnegative - as they are in [6]. To prove (2.10) we shall need the following Tauberian theorem for Laplace transforms due to Von Stachó, see Doetsch [7, p. 277].

Tauberian Theorem. Let $u(t)$ be defined for $t>0$ and let $u(t)$ be continuous for $t \geqq T_{0} \geqq 0$. Let

$$
\bar{u}(s)=\int_{0}^{\infty} e^{-s t} u(t) d t
$$

converge for some $\operatorname{Re} s=\sigma>0$. For a fixed $X>\sigma$ let the integral

$$
\int_{-\infty}^{\infty} e^{i t y} \bar{u}(X+i y) d y
$$

converge uniformly for $t \geqq T_{0}$. Let $\bar{u}(s)$ be analytic for $\operatorname{Re}\{s\} \geqq 0$ with the exception of the point $s=0$ in whose neighborhood $\bar{u}(s)$ is expandable in a series of the form

$$
\bar{u}(s)=\frac{1}{s}\left(c_{0}+d_{0} s^{1 / 2}+c_{1} s+d_{1} s^{3 / 2}+c_{2} s^{2}+d_{2} s^{5 / 2}+\cdots\right) .
$$

In the strip $0 \leqq x \leqq X$, let $\bar{u}(x+i y) \rightarrow 0$ as $|y| \rightarrow \infty$ uniformly in $x$. Let the derivatives $\bar{u}^{\prime}(i y), \bar{u}^{\prime \prime}(i y), \cdots, \bar{u}^{(n)}(i y)(n \geqq 1)$ approach zero as $|y| \rightarrow \infty$, and let the integrals

$$
\int_{Y_{1}}^{\infty}\left|\bar{u}^{(n+1)}(i y)\right| d y, \quad \int_{-\infty}^{-Y_{2}}\left|\bar{u}^{(n+1)}(i y)\right| d y
$$

where $Y_{1}, Y_{2}>0$, converge. Then

$$
\lim _{t \rightarrow \infty} t^{n}\left[u(t)-c_{0}-\frac{1}{\pi \sqrt{ } t} \sum_{i=0}^{n}(-1)^{i} d_{i} \frac{\Gamma\left(j+\frac{1}{2}\right)}{t^{j}}\right]=0 .
$$

The following six lemmas verify that $u(t ; b)$ satisfies the hypotheses of the preceding Tauberian theorem.

Lemma 5.1. Let (2.1) and (2.6) be satisfied. Then

$$
\bar{u}(s ; b)=\int_{0}^{\infty} e^{-s t} u(t ; b) d t
$$


converges for $\operatorname{Re}\{s\}>\gamma$ by Lemma 4.1. Moreover, $\bar{u}(s ; b)$ is analytic for $\operatorname{Re}\{s\} \geqq 0, s \neq 0$, and may be represented there by

$$
\bar{u}(s ; b)=\frac{u_{0}}{s+\frac{1}{\pi a} I_{1}(s)}-\frac{1}{\pi} \frac{I_{2}(s)}{s+\frac{1}{\pi a} I_{1}(s)},
$$

where

$$
I_{i}(s)=\int_{0}^{\infty} \frac{h_{i}(x)}{s+\frac{b}{a} x^{2}} d x \quad(i=1,2) .
$$

Proof. By Lemma $4.1 u(t ; b)$ and $u^{\prime}(t ; b)$ have Laplace transforms. Taking the Laplace transform of both sides of $(3.10)$, with $u(t)$ replaced by $u(t ; b)$ in (3.10), it follows readily from Lemmas 4.1, 4.2 and the convolution theorem for Laplace transforms that for $\operatorname{Re}\{s\}>\gamma$

$$
\left\{s+\frac{1}{\pi a} I_{1}(s)\right\} \bar{u}(s ; b)=u_{0}-\frac{1}{\pi} I_{2}(s) .
$$

The change of variables $x^{2} \rightarrow x$ in (5.3) yields

$$
\frac{2 b}{a} I_{i}(s)=\int_{0}^{\infty} \frac{1}{\frac{a}{b} s+x} \frac{h_{i}\left(x^{\frac{2}{2}}\right)}{x^{\frac{1}{2}}} d x \quad(i=1,2) .
$$

The right-hand side of (5.5) is seen to be the Stieltjes transform of $h_{i}\left(x^{\frac{1}{3}}\right) / x^{\frac{3}{3}}$ evaluated at $a s / b$. From (4.4) we have easily $h_{i}\left(x^{\frac{1}{3}}\right) / x^{\frac{1}{t}} \varepsilon L_{1}(0, \infty)$, and therefore by an elementary theorem for Stieltjes transforms (see WIDDER [8, p. 327]) it follows that $I_{i}(s)$ is an analytic function of $s$ in the cut plane omitting the negative real axis. It only remains to show that $s+I_{1}(s) / \pi a \neq 0$ for $\operatorname{Re}\{s\} \geqq 0, s \neq 0$. But from (5.5), with $s=\sigma+i \tau$,

$$
\operatorname{Re}\left\{\frac{2 b}{a} I_{1}(s)\right\}=\int_{0}^{\infty} \frac{\frac{a}{b} \sigma+x}{\left(\frac{a}{b} \sigma+x\right)^{2}+\left(\frac{a}{b} \tau\right)^{2}} \frac{h_{1}\left(x^{\frac{1}{2}}\right)}{x^{\frac{1}{2}}} d x,
$$

and this, together with (2.6), implies that $\operatorname{Re}\left[s+I_{1}(s) / \pi a\right\}>0$ for $\operatorname{Re}\{s\} \geqq 0$, $s \neq 0$. The result now follows immediately from (5.4) by analytic continuation.

Lemma 5.2. Let (2.1) be satisfied. Then for all s not on the negative real axis

$$
I_{i}^{(n)}(s)=(-1)^{n} n ! \int_{0}^{\infty} \frac{h_{i}(\xi)}{\left[s+\frac{b}{a} \xi^{\xi^{2}}\right]^{n+1}} d \xi \quad(i=1,2 ; n=0,1, \cdots) .
$$

If $s=x+i y$, then

$$
\left|I_{i}^{(n)}(s)\right| \leqq \frac{K_{4} n !}{|y|^{n+1}} \quad(i=1,2 ;-\infty<x<\infty,|y|>0),
$$


where

$$
K_{4}=\max _{i=1,2} \int_{0}^{\infty}\left|h_{i}(\xi)\right| d \xi .
$$

Proof. Referring to the change of variable (5.5), (5.7) is a well known result on Stieltjes transforms (see [8, p. 328]). Using (4.4), the inequality (5.8) follows directly from (5.7), (5.9).

Lemma 5.3. Let (2.1) and (2.6) be satisfied, and let $s=x+i y$. Then

$$
\left|s+\frac{1}{\pi a} I_{1}(s)\right| \geqq|y|-\frac{K_{4}}{\pi a} \frac{1}{|y|} \quad(-\infty<x<\infty,|y|>0),
$$

and $\bar{u}(s ; b)$ is analytic and is represented by $(5.2)$ in the set

$$
S:\{\operatorname{Re}\{s\} \geqq 0, s \neq 0\} \cup\left\{\operatorname{Re}\{s\}<0,|y|>\left[\frac{K_{4}}{\pi a}\right]^{\frac{1}{2}}\right\} .
$$

Proof. Inequality (5.10) is an obvious consequence of $(5.8 ; n=0)$. Lemma 5.1 and analytic continuation complete the proof.

Lemma 5.4. Let (2.1) and (2.6) be satisfied, and let $s=x+i y$. Then for $n=0$, $1, \cdots$

$$
\bar{u}^{(n)}(s ; b)=O\left(|y|^{-n-1}\right) \quad(|y| \rightarrow \infty)
$$

uniformly with respect to $x$.

Proof. Define

$$
p(s)=s+\frac{1}{\pi a} I_{1}(s)
$$

Then by (5.2)

$$
\bar{u}(s ; b)=\frac{u_{0}}{p(s)}-\frac{1}{\pi} \frac{I_{2}(s)}{p(s)} .
$$

It will be shown that for $n=0,1, \ldots$

$$
\begin{aligned}
& \left(\frac{1}{p(s)}\right)^{(n)}=O\left(|y|^{-n-1}\right) \quad(|y| \rightarrow \infty), \\
& \left(\frac{I_{2}(s)}{p(s)}\right)^{(n)}=O\left(|y|^{-n-2}\right) \quad(|y| \rightarrow \infty),
\end{aligned}
$$

uniformly with respect to $x$, which combined with (5.14) yields (5.12).

Clearly (5.10) implies (5.15; $n=0$ ). But from (5.8), (5.13)

$$
\left\{\begin{aligned}
&\left|p^{\prime}(s)\right| \leqq 1+\frac{K_{4}}{\pi a} \frac{1}{|y|^{2}} \quad(|y|>0), \\
&\left|p^{(n)}(s)\right| \leqq \frac{K_{4}}{\pi a} \frac{n !}{|y|^{n+1}} \quad(|y|>0 ; n=2,3, \cdots) .
\end{aligned}\right.
$$


Hence from

$$
\left(\frac{1}{p(s)}\right)^{\prime}=-\frac{p^{\prime}(s)}{p^{2}(s)}, \quad\left(\frac{1}{p(s)}\right)^{(2)}=2 \frac{\left(p^{\prime}(s)\right)^{2}}{p^{3}(s)}-\frac{p^{(2)}(s)}{p^{2}(s)} \quad\left(|y|>\left[\frac{K_{4}}{\pi a}\right]^{\frac{1}{2}}\right)
$$

and from $(5.15 ; n=0)$ just established, together with (5.17), we have (5.15; $n=1,2)$.

The Leibniz formula

$$
(\xi(s) \eta(s))^{(n)}=\sum_{r=0}^{n}\left(\begin{array}{c}
n \\
r
\end{array}\right) \xi^{(r)}(s) \eta^{(n-r)}(s) \quad(n=0,1, \cdots)
$$

easily implies

$$
\left(\frac{1}{p(s)}\right)^{(n)}=-\frac{n}{p(s)} p^{\prime}(s)\left(\frac{1}{p(s)}\right)^{(n-1)}-\frac{1}{p(s)} \sum_{r=2}^{n}\left(\begin{array}{c}
n \\
r
\end{array}\right) p^{(r)}(s)\left(\frac{1}{p(s)}\right)^{(n-r)}
$$

for $n=2,3, \cdots,|y|>\left[K_{4} / \pi a\right]^{\frac{1}{2}}$. An obvious induction using $(5.15 ; n=0)$ and (5.17), (5.19) establishes (5.15; $n=3,4, \cdots)$.

Again, from (5.18) one has

$$
\left(\frac{I_{2}(s)}{p(s)}\right)^{(n)}=\sum_{r=0}^{n}\left(\begin{array}{l}
n \\
r
\end{array}\right) I_{2}^{(r)}(s)\left(\frac{1}{p(s)}\right)^{(n-r)}
$$

for $n=0,1, \cdots,|y|>\left[K_{4} / \pi a\right]^{\frac{1}{2}}$, which, together with (5.8), (5.15), easily yields (5.16).

Lemma 5.5. Let (2.1) and (2.6) be satisfied. Let $X, T_{0}>0$ be fixed. Then the integral

$$
\int_{-\infty}^{\infty} e^{i t y} \bar{u}(X+i y ; b) d y
$$

converges uniformly for $t \geqq T_{0}$.

Proof. Let $\epsilon>0$. It suffices to show that there exists an $\Omega(\epsilon)>0$ such that

$$
\left|\int_{\omega_{1}}^{\omega_{3}} e^{i t y} \bar{u}(X+i y ; b) d y\right| \leqq \frac{\epsilon}{2}, \quad\left|\int_{-\omega_{3}}^{-\omega_{\star}} e^{i t y} \bar{u}(X+i y ; b) d y\right| \leqq \frac{\epsilon}{2}
$$

for all $\omega_{3} \geqq \omega_{1} \geqq \Omega(\epsilon), \omega_{3} \geqq \omega_{4} \geqq \Omega(\epsilon), t \geqq T_{0}$. Here we establish the first inequality of (5.22); the details for the second one are entirely similar.

From (5.2) one has for any $\omega_{2} \geqq \omega_{1}>0$

$$
\int_{\omega_{1}}^{\omega_{2}} e^{i t y} \bar{u}(X+i y ; b) d y=R_{1}+R_{2}
$$

where

$$
\begin{aligned}
& R_{1}=\int_{\omega_{1}}^{\omega_{2}} e^{i t y}\left\{\frac{u_{0}}{X+i y+\frac{1}{\pi a} I_{1}(X+i y)}\right\} d y, \\
& R_{2}=-\frac{1}{\pi} \int_{\omega_{1}}^{\omega_{2}} e^{i t y}\left\{\frac{I_{2}(X+i y)}{X+i y+\frac{1}{\pi \alpha} I_{1}(X+i y)}\right\} d y .
\end{aligned}
$$


Integrating $R_{1}$ by parts there results

$$
\begin{aligned}
R_{1}=\frac{u_{0}}{i t}\left\{e^{i t y} \frac{1}{X+i y+\frac{1}{\pi a} I_{1}(X+i y)}\right\} & \left.\right|_{y=\omega_{2}} ^{y=\omega_{2}} \\
& +\frac{u_{0}}{t} \int_{\omega_{1}}^{\omega_{2}} e^{i t y} \frac{1+\frac{1}{\pi a} I_{1}^{\prime}(X+i y)}{\left[X+i y+\frac{1}{\pi a} I_{1}(X+i y)\right]^{2}} d y .
\end{aligned}
$$

Using (5.8), (5.10) one has for $\omega_{2} \geqq \omega_{1} \geqq\left[K_{4} / \pi a\right]^{\frac{1}{2}}, t \geqq T_{0}$,

$$
\left|R_{1}\right| \leqq \frac{\left|u_{0}\right|}{T_{0}}\left\{\frac{1}{\omega_{2}-\frac{K_{4}}{\pi a \omega_{2}}}+\frac{1}{\omega_{1}-\frac{K_{4}}{\pi a \omega_{1}}}\right\}+\frac{\left|u_{0}\right|}{T_{0}} \int_{\omega_{1}}^{\omega_{2}} \frac{1+\frac{K_{4}}{\pi a} \frac{1}{y^{2}}}{\left[y-\frac{K_{4}}{\pi a y}\right]^{2}} d y
$$

from which there clearly exists an $\Omega_{1}(\epsilon)>0$ such that

$$
\left|R_{1}\right| \leqq \frac{1}{4} \epsilon \quad\left(\omega_{2} \geqq \omega_{1} \geqq \Omega(\epsilon), t \geqq T_{0}\right) .
$$

Turning to $R_{2}$, one has from (5.8), (5.10) for $\omega_{2} \geqq \omega_{1} \geqq\left[K_{4} / \pi a\right]^{\frac{1}{2}}$ and all $t$

$$
\left|R_{2}\right| \leqq \frac{1}{\pi} \int_{\omega_{1}}^{\omega_{2}} \frac{\frac{K_{4}}{y}}{y-\frac{K_{4}}{\pi a y}} d y
$$

from which it is evident that there exists an $\Omega_{2}(\epsilon)>0$ such that

$$
\left|R_{2}\right| \leqq \frac{1}{4} \epsilon \quad\left(\omega_{2} \geqq \omega_{1} \geqq \Omega_{2}(\epsilon)\right) .
$$

Hence, taking $\Omega(\epsilon)=\max \left[\Omega_{1}(\epsilon), \Omega_{2}(\epsilon)\right]$ and combining (5.23), (5.24), (5.25), we obtain the first inequality of (5.22).

Lemma 5.6. Let (2.1), (2.4), (2.6), (2.7), (2.8) be satisfied. Then $\bar{u}(s ; b)$ is expandable, in a neighborhood of $s=0$, in a series of the form

$$
\bar{u}(s ; b)=\frac{1}{s}\left(c_{1} s+d_{1} s^{3 / 2}+c_{2} s^{2}+d_{2} s^{5 / 2}+\cdots\right) .
$$

The coefficients $c_{1}$ and $c_{2}$ are given by

$$
\left\{\begin{aligned}
c_{1}= & -a \frac{H_{2}(0)}{H_{1}(0)} \\
c_{2}=-\left[2 a u_{0} \frac{H_{1}^{\prime}(0)}{H_{1}^{2}(0)}+\frac{1}{2} \frac{a^{2}}{b}\left(2 \frac{\left[H_{1}^{\prime}(0)\right]^{2} H_{2}(0)}{H_{1}^{3}(0)}\right.\right. & \left.\left.-\frac{H_{1}^{\prime \prime}(0) H_{2}(0)+2 H_{1}^{\prime}(0) H_{2}^{\prime}(0)}{H_{1}^{2}(0)}+\frac{H_{2}^{\prime \prime}(0)}{H_{2}(0)}\right)\right]
\end{aligned}\right.
$$

and $d_{1}, d_{2}$ are given by (2.13), where the $H_{i}^{(n)}(0)$ are given by (2.14). 
It is important to observe that, in the notation of the expansion of $\bar{u}(s)$ in the Tauberian Theorem, $c_{0}=d_{0}=0$.

Proof. As the Stieltjes transforms (5.3), (5.5) are not defined when $s$ is a negative real number, the representation $(5.2)$ of $\bar{u}(s ; b)$ is not convenient for the proof of this lemma. Therefore we first obtain another representation, (5.32), for $\bar{u}(s ; b)$.

Define

$$
\left\{\begin{array}{l}
H_{1}(z)=\int_{-\infty}^{\infty} \int_{-\infty}^{\infty} \exp \{-z|x-y|\} \alpha(x) \eta(y) d x d y \\
H_{2}(z)=\int_{-\infty}^{\infty} \int_{-\infty}^{\infty} \exp \{-z|x-y|\} \alpha(x) f(y) d x d y
\end{array} \quad(\operatorname{Re}\{z\}>0) .\right.
$$

The existence of these functions follows readily from (2.1) and the Parseval and convolution theorems. In particular, using

$$
\mathscr{F}\{\exp \{-z|x|\}\}=\int_{-\infty}^{\infty} \exp \{-i x y-z|y|\} d y=\frac{2 z}{z^{2}+x^{2}} \quad(\operatorname{Re}\{z\}>0),
$$

one has

$$
\left\{\begin{array}{l}
H_{1}(z)=\frac{1}{\pi} \int_{-\infty}^{\infty} \frac{z}{z^{2}+y^{2}} \hat{\eta}(y) \hat{\alpha}(-y) d y \\
H_{2}(z)=\frac{1}{\pi} \int_{-\infty}^{\infty} \frac{z}{z^{2}+y^{2}} \hat{f}(y) \hat{\alpha}(-y) d y
\end{array} \quad(\operatorname{Re}\{z\}>0),\right.
$$

which by an elementary change of variable may be written as

$$
H_{i}(z)=\frac{2}{\pi} \int_{0}^{\infty} \frac{z}{z^{2}+y^{2}} h_{i}(y) d y \quad(i=1,2 ; \operatorname{Re}\{z\}>0) .
$$

It follows, as in the proof of Lemma 5.1, from (4.4), (5.30), and an elementary theorem for Stieltjes transforms that the $H_{i}(z)$ are analytic for $\operatorname{Re}\{z\}>0$. Comparing (5.30) with (5.3) one has

$$
I_{i}(s)=\frac{\pi}{2}\left(\frac{a}{b}\right)^{\frac{1}{2}} \frac{1}{s^{\frac{1}{2}}} H_{i}\left(\left(\frac{a}{b}\right)^{\frac{1}{2}} s^{\frac{1}{2}}\right) \quad(i=1,2)
$$

for all $s$ in the cut plane omitting the negative real axis. Combining (5.2), (5.31) yields

$$
\bar{u}(s ; b)=\frac{u_{0} s^{\frac{1}{2}}}{s^{\frac{3}{2}}+\frac{1}{2(a b)^{\frac{1}{2}}} H_{1}\left(\left(\frac{a}{b}\right)^{\frac{1}{2}} s^{\frac{1}{2}}\right)}-\frac{1}{2}\left(\frac{a}{b}\right)^{\frac{1}{2}} \frac{H_{2}\left(\left(\frac{a}{b}\right)^{\frac{1}{2}} s^{\frac{1}{2}}\right)}{s^{\frac{3}{2}}+\frac{1}{2(a b)^{\frac{1}{2}}} H_{1}\left(\left(\frac{a}{b}\right)^{\frac{1}{2}} s^{\frac{1}{2}}\right)},
$$

valid for all $s$ such that $\operatorname{Re}\{s\} \geqq 0, s \neq 0$. In fact, (5.32) is clearly valid in the larger set $S$ of (5.11), although this fact will not be used here. 
Let

$$
U(z)=\frac{u_{0} z}{z^{3}+\frac{1}{2(a b)^{\frac{1}{2}}} H_{1}\left(\left(\frac{a}{b}\right)^{\frac{1}{2}} z\right)}-\frac{1}{2}\left(\frac{a}{b}\right)^{\frac{1}{2}} \frac{H_{2}\left(\left(\frac{a}{b}\right)^{\frac{1}{2}} z\right)}{z^{3}+\frac{1}{2(a b)^{\frac{1}{2}}} H_{1}\left(\left(\frac{a}{b}\right)^{\frac{1}{2}} z\right)} .
$$

Then from (5.32), (5.33), $\bar{u}(s ; b)=U\left(s^{\frac{1}{2}}\right)$.

We now show that $U(z)$ is analytic for $|z|$ sufficiently small. Using (2.4), (2.7) it will first be shown that the $H_{i}(z)$ are analytic for $\operatorname{Re}\{z\}>-\lambda$. Let $\delta_{1}$ satisfy $0<\delta_{1}<\lambda$. Then an elementary calculation using (2.4), (2.7) implies the existence of a constant $K_{5}>0$ such that

$$
\int_{-\infty}^{\infty}|\alpha(x) \eta(x-g)| d x \leqq K_{5} e^{-\delta_{1}|g|} \quad(-\infty<g<\infty)
$$

It easily follows from (5.28), (5.34), the change of variable $x-y=g$, and Fubini's theorem that

$$
H_{1}(z)=\int_{-\infty}^{\infty} e^{-z|g|}\left\{\int_{-\infty}^{\infty} \alpha(x) \eta(x-g) d x\right\} d g \quad\left(\operatorname{Re}\{z\}>-\delta_{1}\right) .
$$

This yields the analyticity of $H_{1}(z)$ for $\operatorname{Re}\{z\}>-\lambda$, since from (5.34) it follows that (5.35) converges uniformly with respect to $z$ for $\operatorname{Re}\{z\} \geqq-\delta_{2}>-\delta_{1}$. The same argument shows that $H_{2}(z)$ is analytic for $\operatorname{Re}\{z\}>-\lambda$. Since $H_{1}(0) \neq 0$, by (2.8), it follows from (5.33) and the analyticity of the $H_{\imath}(z)$ that $U(z)$ is analytic for $|z|$ sufficiently small.

Therefore by analytic continuation (5.32) is also valid in a sufficiently small neighborhood of $s=0$, and thus $\bar{u}(s ; b)$ is expandable there in a series of the form (5.26). The formulae (2.13), (2.14), (5.27) follow in a routine but tedious fashion. This completes the proof of Lemma 5.6.

Remark. If $H_{1}(0)=0$, but $H_{1}^{\prime}(0) \neq 0$, the preceding analysis is still valid except that the expansion (5.26) would then have a $d_{0} \neq 0$ term, in general. The asymptotic behavior of $u(t ; b)$ would be modified accordingly. Note, however, that in connection with the reactor problem $H_{1}(0)=0$ is completely unrealistic.

The assertion (2.10), and hence also (2.11), of Theorem 2 now follows from the Tauberian Theorem and the preceding lemmas. It only remains to establish (2.12). From (2.11) one has immediately that

$$
u(t ; b)=O\left(t^{-\frac{3}{2}}\right) \quad(t \rightarrow \infty),
$$

which, together with the continuity of $u(t ; b)$, implies

$$
\int_{0}^{\infty}|u(t ; b)| d t<\infty
$$

From (2.2), (4.15) one has 


$$
\left|T_{H}(x, t ; b)\right| \leqq K_{6} t^{-\frac{1}{2}} \quad(-\infty<x<\infty, 0 \leqq t<\infty)
$$

and

$$
\begin{aligned}
|\tilde{T}(x, t ; b)| \leqq \frac{1}{a} K_{6} \int_{0}^{t}(t-\tau)^{-\frac{1}{2}}|u(\tau ; b)| d \tau & \\
& (-\infty<x<\infty, 0 \leqq t<\infty),
\end{aligned}
$$

where

$$
K_{6}=\left(4 \pi \frac{b}{a}\right)^{-\frac{1}{2}} \max \left(\int_{-\infty}^{\infty}|f(\xi)| d \xi, \int_{-\infty}^{\infty}|\eta(\xi)| d \xi\right) .
$$

It is an elementary exercise to show that (5.36), (5.37), (5.39) imply

$$
\tilde{T}(x, t ; b)=O\left(t^{-\frac{1}{3}}\right) \quad(t \rightarrow \infty, \text { uniformly in } x) .
$$

Combining (4.15), (5.38), (5.40) yields (2.12) and completes the proof of Theorem 2 .

6. Proof of Theorem 3. Hypothesis (2.4) clearly implies

$$
\left\{\begin{array}{l}
(i)\left|\int_{-\infty}^{\infty} G(x-\xi, t) f(\xi) d \xi\right| \leqq K_{1} \\
(i i)\left|\int_{-\infty}^{\infty} G(x-\xi, t) \eta(\xi) d \xi\right| \leqq K_{1} .
\end{array} \quad(-\infty<x<\infty, 0 \leqq t<\infty)\right.
$$

From (6.1i) it is clear that the uniqueness asserted in Theorem 3 is equivalent to the assertion (see (3.1)-(3.5)) that there is at most one solution of

$$
\left\{\begin{aligned}
\frac{d u}{d t} & =-\int_{-\infty}^{\infty} \alpha(x) \tilde{T}(x, t) d x-\int_{-\infty}^{\infty} \int_{-\infty}^{\infty} G(x-\xi, t) f(\xi) \alpha(x) d \xi d x \\
a \frac{\partial \tilde{T}}{\partial t} & =b \frac{\partial^{2} \tilde{T}}{\partial x^{2}}+\eta(x) u
\end{aligned}\right.
$$

satisfying

$$
u(0)=u_{0}, \quad \tilde{T}(x, 0) \equiv 0 \quad(-\infty<x<\infty)
$$

such that

$$
\text { (i) } \int_{0}^{\infty}|u(t)| d t<\infty, \quad \text { (ii) } \sup _{\substack{-\infty<x<\infty \\ 0 \leq t<\infty}}|\tilde{T}(x, t)|<\infty .
$$

Suppose there are two solutions $u_{j}(t), T_{j}(x, t)(j=1,2)$ of $(6.2)$, (6.3) that satisfy (6.4). Define

$$
T^{*}(x, t)=\frac{1}{a} \int_{0}^{t} \int_{-\infty}^{\infty} G(x-\xi, t-\tau) \eta(\xi) u_{j}(\tau) d \xi d \tau \quad(j=1,2) .
$$


Then it follows readily from (6.1ii), (6.4i) that $\left|T_{i}^{*}(x, t)\right| \leqq K_{7}(j=1,2)$, for some positive constant $K_{z}$. Thus both $\tilde{T}_{i}$ and $T_{i}^{*}$ are bounded solutions of

$$
a \frac{\partial T}{\partial t}=b \frac{\partial^{2} T}{\partial x^{2}}+\eta(x) u_{i}
$$

the first by hypothesis and the second by the same classical result referred to in the proof of Theorem 1. Since

$$
\tilde{T}_{j}(x, 0)=T_{i}^{*}(x, 0) \equiv 0 \quad(-\infty<x<\infty),
$$

it follows by a well known uniqueness theorem for the heat equation that

$$
\tilde{T}_{j}(x, t) \equiv T_{i}^{*}(x, t) \quad(j=1,2) .
$$

Replacing $u, \tilde{T}$ in (6.2) by $u_{j}, \tilde{T}_{i}$ and using (6.5), (6.6) we obtain, with the aid of Fubini's theorem,

$$
\begin{aligned}
\frac{d u_{j}}{d t}=-\frac{1}{a} \int_{0}^{t}\left\{\int_{-\infty}^{\infty}\right. & \left.\int_{-\infty}^{\infty} G(x-\xi, t-\tau) \eta(\xi) \alpha(x) d \xi d x\right\} u_{j}(\tau) d \tau \\
& -\int_{-\infty}^{\infty} \int_{-\infty}^{\infty} G(x-\xi, t) f(\xi) \alpha(x) d \xi d x \quad(j=1,2)
\end{aligned}
$$

where by hypothesis $u_{1}(0)=u_{2}(0)=u_{0}$. However, (6.7) is the same as (3.7). Therefore, by Lemma 4.3, it follows that $u_{1}(t) \equiv u_{2}(t)$. But then (6.5), (6.6) imply that $\tilde{T}_{1}(x, t) \equiv \tilde{T}_{2}(x, t)$, which completes the proof of Theorem 3 .

7. Proof of Theorem 4. To show that $u=v(t), T=\pi(x, t)$ (given by (2.17)) is a solution of (1.3), (1.2) is an elementary calculation and is omitted here.

To prove uniqueness suppose there are two solutions $u_{j}(t), T_{i}(x, t)(j=1,2)$ of (1.3), (1.2). Then by hypothesis the functions

$$
y_{j}(t)=\int_{-\infty}^{\infty} \alpha(x) T_{j}(x, t) d x \quad(j=1,2)
$$

exist for $0 \leqq t<\infty$, and from (1.3)

$$
\frac{d u_{j}}{d t}=-y_{j} \quad(j=1,2)
$$

It follows from (7.1) and the hypothesis that

$$
y_{1}(0)=y_{2}(0)=(\alpha, f) \text {. }
$$

The hypothesis and (1.3) also imply that

$$
\alpha(x) \frac{\partial T_{i}}{\partial t}=\frac{1}{a} \alpha(x) \eta(x) u_{i} \quad(j=1,2),
$$


and as $\alpha(x) \eta(x) \varepsilon L_{1}(-\infty, \infty)$, by (2.1), it follows that

$$
\int_{-\infty}^{\infty} \alpha(x) \frac{\partial T_{i}}{\partial t}(x, t) d x
$$

converges uniformly with respect to $t$ in any finite interval $0 \leqq t \leqq T_{0}$. Hence we have from (7.1), (7.4)

$$
\frac{d y_{i}}{d t}=\frac{1}{a}(\alpha, \eta) u_{j} \quad(j=1,2),
$$

and by hypothesis

$$
u_{1}(0)=u_{2}(0)=u_{0} .
$$

The uniqueness theorem for linear systems of ordinary differential equations applied to the system (7.2), (7.5) now yields, because of (7.3), (7.6),

$$
u_{1}(t) \equiv u_{2}(t), \quad y_{1}(t) \equiv y_{2}(t) \quad(0 \leqq t<\infty),
$$

and this, together with (1.3) and $T_{1}(x, 0)=T_{2}(x, 0)=f(x)$, yields

$$
T_{1}(x, t) \equiv T_{2}(x, t) \quad(-\infty<x<\infty, 0 \leqq t<\infty),
$$

thus completing the proof of Theorem 4 .

8. Proof of Theorem 5. It follows immediately from (2.17) that $v(t)$ satisfies

$$
\frac{d v}{d t}=-\frac{1}{a}(\alpha, \eta) \int_{0}^{t} v(\tau) d \tau-(\alpha, f) .
$$

An application of the Parseval theorem yields

$$
\frac{d v}{d t}=-\frac{1}{\pi a}\left(\int_{0}^{\infty} h_{1}(x) d x\right) \int_{0}^{t} v(\tau) d \tau-\frac{1}{\pi} \int_{0}^{\infty} h_{2}(x) d x,
$$

where the $h_{i}(x)$ are given by (3.11).

Let $0<T_{1}<\infty$ with $T_{1}$ fixed. Let $\epsilon>0$ be given. Then from (2.6), (4.4) it follows that there exists a $b(\epsilon)>0$ such that

$$
\left\{\begin{aligned}
&\left|1-\frac{\int_{0}^{\infty} h_{1}(x) d x}{\int_{0}^{\infty} \exp \left[-\frac{b}{a} x^{2}(t-\tau)\right] h_{1}(x) d x}\right| \leqq \epsilon \\
&(0<b \leqq b(\epsilon) ;\left.0 \leqq \tau \leqq t \leqq T_{1}\right) \\
&\left|\int_{0}^{\infty}\left\{\exp \left[-\frac{b}{a} x^{2} t\right]-1\right\} h_{2}(x) d x\right| \leqq \epsilon \\
&\left(0<b \leqq b(\epsilon) ; \quad 0 \leqq t \leqq T_{1}\right) .
\end{aligned}\right.
$$


Thus, from (3.10), (8.1), (8.2), and Lemma 4.2, we have

$$
\begin{aligned}
\frac{d}{d t}[u(t ; b)-v(t)]= & -\frac{1}{\pi} \int_{0}^{\infty}\left[\exp \left\{-\frac{b}{a} x^{2} t\right\}-1\right] h_{2}(x) d x \\
-\frac{1}{\pi a} \int_{0}^{t} & {\left[\int_{0}^{\infty} \exp \left\{-\frac{b}{a} x^{2}(t-\tau)\right\} h_{1}(x) d x\right]\{u(\tau ; b)-v(\tau)} \\
& \left.+v(\tau)\left[1-\frac{\int_{0}^{\infty} h_{1}(x) d x}{\int_{0}^{\infty} \exp \left\{-\frac{b}{a} x^{2}(t-\tau)\right\} h_{1}(x) d x}\right]\right\} d \tau
\end{aligned}
$$

for $0<b \leqq b(\epsilon), 0 \leqq t \leqq T_{1}$. Noting that $v(t)$ is bounded on $0 \leqq t \leqq \infty$, it follows from (8.2), (8.3) that there exists a constant $K_{8}>0$, independent of $\epsilon$, such that

$$
\left|\frac{d}{d t}[u(t ; b)-v(t)]\right| \leqq K_{8} \int_{0}^{t}|u(\tau ; b)-v(\tau)| d \tau+K_{8}(1+t) \epsilon
$$

for $0<b \leqq b(\epsilon), 0 \leqq t \leqq T_{1}$. Integrating (8.4) and applying a well known inequality yields

$$
|u(t ; b)-v(t)| \leqq \epsilon K_{8}\left(T_{1}+\frac{1}{2} T_{1}^{2}\right) e^{K_{8} T_{1} t} \quad\left(0<b \leqq b(\epsilon) ; \quad 0 \leqq t \leqq T_{1}\right) .
$$

The result (2.18) now follows directly from (8.5).

From (2.17) one readily has

$$
\pi(x, t)=f(x)+\frac{1}{a} \eta(x) \int_{0}^{t} v(\tau) d \tau
$$

and from (4.15)

$$
\begin{aligned}
T(x, t ; b)=\int_{-\infty}^{\infty} G(x-\xi, t) f(\xi) d \xi & \\
& +\frac{1}{a} \int_{0}^{t} \int_{-\infty}^{\infty} G(x-\xi, t-\tau) \eta(\xi) u(\tau ; b) d \xi d \tau .
\end{aligned}
$$

The result (2.19) now follows directly from (2.18), (8.6), (8.7), and hypothesis (2.3) on applying a classical result for the heat equation. This completes the proof of Theorem 5 .

\section{Appendix}

As the following relaxation of hypotheses may be of interest and can be obtained with little additional effort, we include it here.

In Theorem 1 (2.2) can be dropped entirely and (2.4) replaced by bounded- 
ness of $\eta(x)$ only. Lemmas 4.1, 4.2, 4.3 are obviously not affected by this change. Conditions (2.1), (2.3), (2.5) are enough to insure that the second equations of (1.1), (1.2) are satisfied. By Fubini's theorem the following estimate (and another just like it) verifies (4.16) and, therefore, completes the proof of Theorem 1:

$$
\begin{aligned}
\left\{\int_{-\infty}^{\infty}|\alpha(x)|\left[\int_{-\infty}^{\infty} G(x-\xi, t-\tau)|\eta(\xi)| d \xi\right] d x\right\}^{2} \\
\leqq \int_{-\infty}^{\infty}|\alpha(x)|^{2} d x \int_{-\infty}^{\infty}\left\{\int_{-\infty}^{\infty} G(x-\xi, t-\tau)|\eta(\xi)| d \xi\right\}^{2} d x \\
\leqq \int_{-\infty}^{\infty}|\alpha(x)|^{2} d x \int_{-\infty}^{\infty}|\eta(x)|^{2} d x
\end{aligned}
$$

For by Schwarz's inequality (this is a special case of a well known proof appearing in TitchmaRsh, Theory of Fourier Integrals, page 91)

$$
\begin{aligned}
\left\{\int_{-\infty}^{\infty}[G(x-\xi, t-\tau)]^{\frac{1}{2}}[G(x-\xi, t-\tau)]^{\frac{1}{2}}|\eta(\xi)| d \xi\right\}^{2} & \\
& \leqq \int_{-\infty}^{\infty} G(x-\xi, t-\tau)|\eta(\xi)|^{2} d \xi
\end{aligned}
$$

and therefore

$$
\int_{-\infty}^{\infty}\left\{\int_{-\infty}^{\infty} G(x-\xi, t-\tau) \eta(\xi) d \xi\right\}^{2} d x \leqq \int_{-\infty}^{\infty}|\eta(\xi)|^{2} d \xi .
$$

The above estimate is also readily obtained by the convolution and Parseval theorems approach of Lemma 4.3 .

In Theorem 2 (2.4) can be replaced by boundedness of $\eta(x)$ only. Hypothesis (2.2) is redundant; for it is seen to be implied by (2.1), (2.7). Lemmas 5.1-5.5 remain unchanged. In Lemma 5.6 the continuity of

$$
p(g)=\int_{-\infty}^{\infty} \alpha(x) \eta(x-g) d x
$$

and the estimate (5.34) on it may now be established in the following manner. As

$$
p(g+\Delta)-p(g)=\int_{-\infty}^{\infty} \alpha(x)[\eta(x-g-\Delta)-\eta(x-g)] d x
$$

the former follows from

$$
|p(g+\Delta)-p(g)|^{2} \leqq \int_{-\infty}^{\infty}|\alpha(x)|^{2} d x \int_{-\infty}^{\infty}|\eta(x-\Delta)-\eta(x)|^{2} d x
$$

and a well known theorem. To prove the latter we note from (2.7) that there exist constants $K_{9}$ and $x_{0}$ such that

$$
|\alpha(x)|,|\eta(x)| \leqq K_{9} e^{-\lambda|x|} \quad\left(x_{0} \leqq|x|\right) .
$$


Let $n>2$ and fixed. For $g>0$ and sufficiently large one has

$$
\begin{aligned}
& |p(g)| \leqq\left(\int_{-\infty}^{(1 / n) g}+\int_{(1 / n) g}^{(1-1 / n) g}+\int_{(1-1 / n) g}^{\infty}\right)|\alpha(x)||\eta(x-g)| d x \\
& \leqq K_{9} \int_{-\infty}^{(1 / n) g}|\alpha(x)| \exp \{-\lambda|x-g|\} d x \\
& \quad+K_{9}^{2} \int_{(1 / n) g}^{(1-1 / n) g} \exp \{-\lambda|x|\} \exp \{-\lambda|x-g|\} d x \\
& \quad+K_{9} \int_{(1-1 / n) g}^{\infty} \exp \{-\lambda|x|\}|\eta(x-g)| d x \\
& \leqq K_{9}\left\{\int_{-\infty}^{\infty}(|\alpha(x)|+|\eta(x)|) d x+K_{9}\left(1-\frac{2}{n}\right) g \exp \left\{-\frac{1}{n g}\right\}\right\} \exp \left\{-\lambda g\left(1-\frac{1}{n}\right)\right\} .
\end{aligned}
$$

A similar calculation for $-g>0$ and sufficiently large, together with the continuity of $p(g)$, yields (5.34). The remainder of the proofs of Lemma 5.6 and of Theorem 2 is unchanged.

As in Theorem 1, (2.2) can be dropped entirely and (2.4) replaced by boundedness of $\eta(x)$ only in Theorem 5 . The same result for the inhomogeneous heat equation used in the above version of Theorem 1 is all that is required here.

\section{BIBLIOGRAPHY}

[1] Ergen, W. K., Kinetics of the circulating-fuel nuclear reactor, Jour. Appl. Physics, 15 (1954), pp. 702-711.

[2] Ergen, W. K. \& Nohel, J. A., Stability of a continuous medium reactor, to appear in J. Nucl. Energy, Part A: Reactor Science, 10 (1959), pp. 14-18.

[3] Ergen, W. K., Lipkin, H. J. \& Noher, J. A. Applications of Liapounov's second method in reactor dynamics, Jour. Math. and Physics, 36 (1957), pp. 36-48.

[4] Achieser, N. I., Theory of Approximation, New York (1956).

[5] Fonsythe, G. E. \& Rosenbloom, P. C., Numerical Analysis and Partial Differential Equations, New York (1958).

[6] Feller, W., On the integral equation of renewal theory, Annals of Math. Stat., 12 (1941), pp. 243-267.

[7] Doetsch, G., Theorie und Anwendung der Laplace-Transformation, Berlin (1937).

[8] Widder, D. V., The Laplace Transform, Princeton (1946).

\section{Lincoln Laboratory}

Massachusetts Institute of Technology

Lexington, Massachusetts;

Mathematics Research Center, U. S. Army

Madison, Wisconsin;

and

Georgia Institute of Technology

Atlanta, Georgia 